\title{
Lagrangian dynamic SGS model for Stochastic Coherent Adaptive Large Eddy Simulation
}

\author{
Oleg V. Vasilyev†*, Giuliano De Stefano $\$$, \\ Daniel E. Goldstein§, and Nicholas K.-R. Kevlahan†† \\ $\dagger$ Department of Mechanical Engineering, University of Colorado, \\ 427 UCB, CO 80309 Boulder, USA \\ $\ddagger$ Dipartimento di Ingegneria Aerospaziale e Meccanica, Seconda Universitá di Napoli, \\ I 81031 Aversa, Italy \\ $\S$ Northwest Research Associates, Inc., CORA Division, 3380 Mitchell Lane, CO \\ 80301, Boulder, USA \\ $\dagger \dagger$ Department of Mathematics \& Statistics, McMaster University, Hamilton, ON, \\ L8S 4K1, Canada
}

\begin{abstract}
Stochastic Coherent Adaptive Large Eddy Simulation (SCALES) is an extension of Large Eddy Simulation that uses a wavelet filter-based dynamic grid adaptation strategy to solve for the most energetic coherent structures in a turbulent flow field, while modelling the effect of the less energetic ones. A localized dynamic subgrid scale model is needed to fully exploit the ability of the method to track coherent structures. In this paper, new local Lagrangian models based on a modified Germano dynamic procedure, redefined in terms of wavelet thresholding filters, are proposed. These models extend the original path-line formulation of Meneveau et al [J. Fluid Mech., 319, 1996] in two ways: as Lagrangian path-line diffusive and Lagrangian path-tube averaging procedures. The proposed models are tested for freely decaying homogeneous turbulence with initial $R e_{\lambda}=72$. It is shown that the SCALES results, obtained with fewer than $0.4 \%$ of the total non-adaptive nodes required for a DNS with the same wavelet solver, closely match reference DNS data. In contrast to classical LES, this agreement holds not only for large scale global statistical quantities, but also for energy and, more importantly, enstrophy spectra up to the dissipative wavenumber range.
\end{abstract}

\footnotetext{
* To whom correspondence should be addressed (Oleg.Vasilyev@Colorado.edu)
} 


\section{Introduction}

Although turbulence is common in engineering applications, a solution to the fundamental equations that govern turbulent flow still eludes the scientific community. Due to the prohibitively large disparity of spatial and temporal scales, direct numerical simulation (DNS) of turbulent flows of engineering interest are impossible, even with the aid of the fastest supercomputers that exist or will be available in the foreseeable future. Large eddy simulation (LES) is often viewed as a feasible alternative for turbulent flow modeling, e.g., [1]. The main idea behind LES is to resolve only the large-scale motions, while modeling the effect of the unresolved subgrid scale (SGS) eddies.

When dealing with complex turbulent flows, current LES methods rely on, at best, a zonal grid adaptation strategy to minimize the computational cost. This mesh is typically non-adaptive and chosen in a somewhat ad hoc manner to adequately resolve the flow. While an improvement over the use of regular grids, these methods fail to resolve the high wavenumber components of the spatially and temporarily intermittent coherent structures characteristic of turbulent flows, thus neglecting valuable physical information. At the same time, the flow is over-resolved in regions between the coherent structures, which wastes computational resources. Finally, as pointed out by [2], the local filter width in LES implementations is typically proportional to local mesh size, which makes LES results highly dependent on the computational grid.

We have recently introduced a novel approach to turbulent flow simulation, called Stochastic Coherent Adaptive Large Eddy Simulation (SCALES) [3, 4]. This method addresses the above mentioned shortcomings of LES by using a wavelet thresholding filter to dynamically resolve and track the most energetic coherent structures during the simulation, while modeling the effect of the unresolved less energetic modes. We

showed that the residual motions are composed of a small number of coherent modes that dominate the total SGS dissipation and a large number of incoherent modes that, due to their decorrelation with the resolved modes, add little to the total subgrid scale dissipation $[3,5]$. Therefore, in this work, as in much of classical LES, only the coherent part of the unresolved modes is modelled using a deterministic SGS stress model.

The first step towards the construction of SGS models for SCALES was undertaken in [4], where we developed a global dynamic Smagorinsky eddy viscosity model based on the classical Germano procedure redefined in terms of two wavelet thresholding filters. The main drawback of this formulation is the use of a global (spatially non-variable) model coefficient. The use of a global dynamic model unnecessarily limits the SCALES approach to flows with at least one homogeneous direction. This is unfortunate since the dynamic adaptability of SCALES is ideally suited to fully inhomogeneous flows. In this paper a local dynamic model is developed to extend SCALES to fully inhomogeneous turbulent flows. The proposed models are based on two different extensions of the original Lagrangian path-line formulation of [6], namely Lagrangian path-line diffusive and Lagrangian path-tube averaging procedures.

The paper is organized as follows. The SCALES methodology for the numerical 
solution of turbulent flows is reviewed briefly in $\S 2$. The local dynamic Smagorinsky model with Lagrangian path-line/tube averaging is discussed in detail in $\S 3$. The results for the numerical simulations are presented in $\S 4$ and, finally, some concluding remarks are made in $\S 5$.

\section{Stochastic coherent adaptive large eddy simulation}

\subsection{Wavelet Thresholding Filter}

Let us very briefly outline the main features of the wavelet thresholding filter. More details can be found, for instance, in [7]. A velocity field $u_{i}(\mathbf{x})$ can be represented in terms of wavelet basis functions as

$$
u_{i}(\mathbf{x})=\sum_{\mathbf{l} \in \mathcal{L}^{0}} c_{\mathbf{l}}^{0} \phi_{\mathbf{l}}^{0}(\mathbf{x})+\sum_{j=0}^{+\infty} \sum_{\mu=1}^{2^{n}-1} \sum_{\mathbf{k} \in \mathcal{K}^{\mu, j}} d_{\mathbf{k}}^{\mu, j} \psi_{\mathbf{k}}^{\mu, j}(\mathbf{x}),
$$

where $\phi_{\mathbf{l}}^{0}$ and $\psi_{\mathbf{k}}^{\mu, j}$ are $n$-dimensional scaling functions and wavelets of different families and levels of resolution, indexed with $\mu$ and $j$, respectively. One way to think of a wavelet decomposition is as a multi-level or multi-resolution representation of $u_{i}$, where each level of resolution $j$ (except the coarsest one) consists of a family of wavelets $\psi_{\mathbf{k}}^{\mu, j}$ having the same scale but located at different positions. Scaling function coefficients represent the averaged values of the field, while the wavelet coefficients represent the details of the field at different scales.

Wavelet filtering is performed in wavelet space using wavelet coefficient thresholding, which is a nonlinear filter that depends on each flow realization. The wavelet thresholding filter is defined by,

$$
\bar{u}_{i}^{>\epsilon}(\mathbf{x})=\sum_{\mathbf{l} \in \mathcal{L}^{0}} c_{\mathbf{l}}^{0} \phi_{\mathbf{l}}^{0}(\mathbf{x})+\sum_{j=0}^{+\infty} \sum_{\mu=1}^{2^{n}-1} \sum_{\substack{\mathbf{k} \in \mathcal{K}^{\mu, j} \\ \mid d_{\mathbf{k}}^{\mu, j}>>\epsilon\left\|u_{i}\right\|_{\mathrm{WTF}}}} d_{\mathbf{k}}^{\mu, j} \psi_{\mathbf{k}}^{\mu, j}(\mathbf{x}),
$$

where $\epsilon>0$ stands for the non-dimensional (relative) threshold parameter, $\|\cdot\|_{\text {wTF }}$ being the Wavelet Threshold Filtering (WTF) norm that provides the (absolute) dimensional velocity scale in the $i$-th direction. For instance, the (absolute) dimensional scaling can be specified as the $L_{2}$ norm $\left(\left\|u_{i}\right\|_{\mathrm{WTF}}=\left\|u_{i}\right\|_{2}\right)$ or the $L_{\infty} \operatorname{norm}\left(\left\|u_{i}\right\|_{\mathrm{WTF}}=\left\|u_{i}\right\|_{\infty}\right)$. Note that once the WTF-norm $\|\cdot\|_{\text {WTF }}$ is specified, the wavelet thresholding filter (2) is uniquely defined by the nondimensional threshold parameter, $\epsilon$. Also note that for simulation of homogeneous turbulence the same scaling is used in all three directions, e.g. $\left\|u_{i}\right\|_{\mathrm{wTF}}=\|\mathbf{u}\|_{2}$.

The major strength of wavelet filtering is its ability to compress the solution. For turbulent fields, which contain isolated high-energy coherent structures on a low-energy background, most wavelet coefficients are small. Thus, a good approximation can be retained even after discarding a large number of wavelets with small coefficients. Intuitively, in the wavelet decomposition (1), the coefficient $d_{\mathbf{k}}^{\mu, j}$ is small unless $u_{i}$ has significant variation on the level of resolution (scale) $j$, in the immediate vicinity of wavelet $\psi_{\mathbf{k}}^{\mu, j}(\mathbf{x})$. 

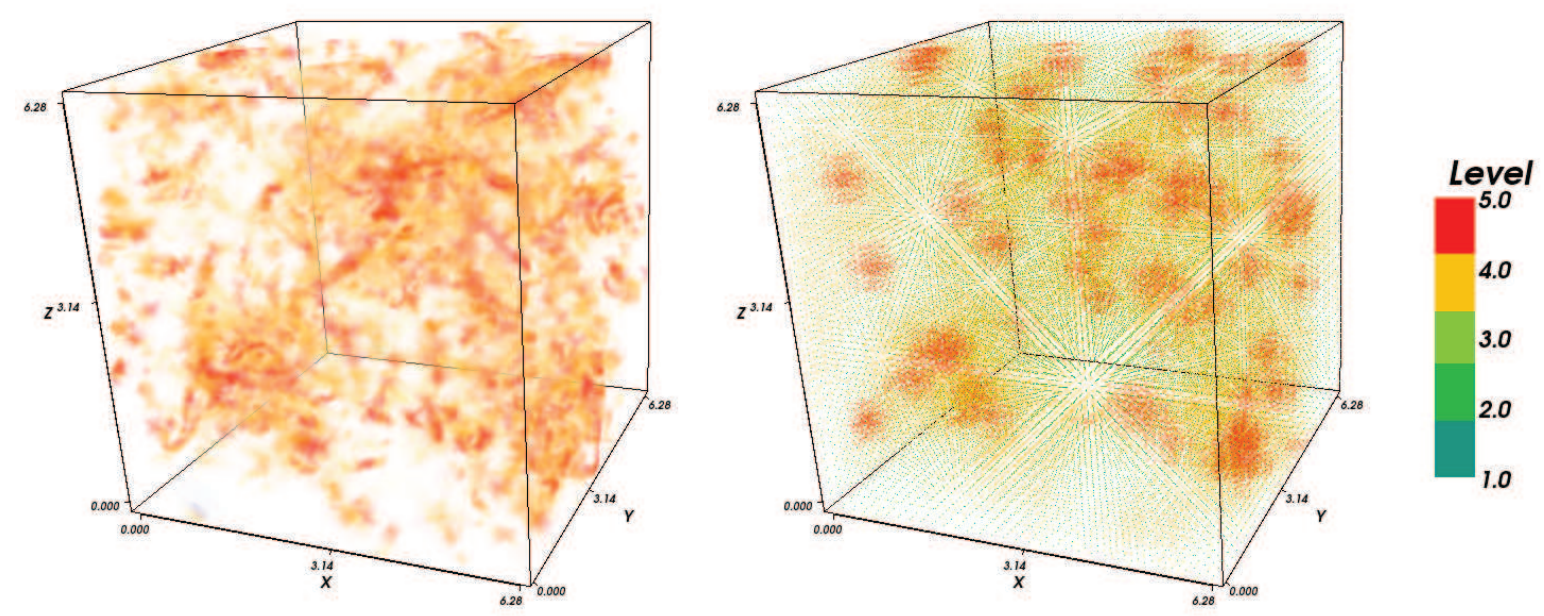

Figure 1. Volume rendered vorticity magnitude (left) and corresponding computational grid (right) for SCALES simulations. Points of different levels of resolution are shown in different color.

\subsection{Wavelet-filtered Navier-Stokes equations}

When applying the wavelet thresholding filter to the Navier-Stokes equations, each variable should be filtered, according to (2), with a corresponding absolute scale. However, this would lead to numerical complications due to the one-to-one correspondence between wavelet locations and grid points. In particular, each variable would be solved on a different numerical grid. In order to avoid this difficulty, the coupled wavelet thresholding strategy is used in the present study. Namely, after constructing the masks of significant wavelet coefficients for each primary variable, the union of these masks results in a global thresholding mask that is used for filtering each term. Note that additional variables, e.g. vorticity or strain rate, can be used to construct the global mask.

Once the global mask is constructed, one can view the wavelet thresholding procedure as local low-pass filtering. Such an interpretation highlights the similarity between SCALES and classical LES approaches. However, the wavelet filter is drastically different from the LES filters because it adapts dynamically to the solution. This generates an adaptive computational grid that tracks the areas of locally significant energy in physical space as illustrated, for instance, in Fig.1 where volume rendered vorticity magnitude and corresponding computational grid used in SCALES simulations are shown.

Therefore, the SCALES equations for incompressible flow, which describe the evolution of the most energetic coherent structures, are obtained by applying the wavelet thresholding filter to the incompressible Navier-Stokes equations:

$$
\frac{\partial \overline{u_{i}}>\epsilon}{\partial x_{i}}=0
$$




$$
\frac{\partial \overline{u_{i}}>\epsilon}{\partial t}+\frac{\partial\left(\overline{u_{i}}>\epsilon \overline{u_{j}}>\epsilon\right.}{\partial x_{j}}=-\frac{1}{\rho} \frac{\partial \bar{p}^{>\epsilon}}{\partial x_{i}}+\nu \frac{\partial^{2} \overline{u_{i}}>\epsilon}{\partial x_{j} \partial x_{j}}-\frac{\partial \tau_{i j}}{\partial x_{j}},
$$

where $\rho, \nu$ are the constant density and kinematic viscosity, and $p$ stands for the pressure. As a result of the filtering process, the unresolved quantities

$$
\tau_{i j}=\bar{u}_{i} u_{j}^{>\epsilon}-\bar{u}_{i}^{>\epsilon} \bar{u}_{j}^{>\epsilon},
$$

commonly referred to as SGS stresses, are introduced. They represent the effect of unresolved (less energetic) coherent and incoherent eddies on the resolved (energetic) coherent vortices. As usual in a LES approach, in order to close Eqs. (4), a closure model is needed to express the unknown SGS stresses in terms of the resolved wavelet-filtered field. Note that analogous to LES with non-uniform filter width $[8,9,10]$ there is a commutation error between wavelet filtering and derivative operators, the effect of which is not considered in this paper. However, a significant number of wavelets below the thresholding level, $\epsilon\left\|u_{i}\right\|_{\mathrm{WTF}}$, are retained due to the adjacent zone and reconstruction check procedures $[11,12,13])$ in the regions of the energetic vortices, which results in a significant reduction of the commutation error.

\subsection{Numerical implementation}

The SCALES methodology is implemented using the adaptive wavelet collocation method (AWCM), e.g. [11]. The wavelet collocation method employs wavelet compression as an integral part of the numerical algorithm such that the solution is obtained with the minimum number of grid points for a given accuracy.

Briefly, the AWCM is an adaptive, variable-order method for solving partial differential equations with localized structures that change their location and scale. Since the computational grid automatically adapts to the solution, both in position and scale, the regions of high gradients or localized structures do not need to be known a priori. Also, the method is based on second-generation wavelets [14], which allow the order of the wavelet (and hence of the numerical method) to be varied easily. The method has a computational complexity $O(N)$, where $N$ is the number of wavelets* retained in the calculation, i.e. those wavelets with significant coefficients plus nearest neighbors. As far as computational cost is concerned, it should be noted that the adaptive wavelet collocation code is about two to three times slower per grid point than the non-adaptive finite difference code, so a compression factor of 260 (that is retaining $0.385 \%$ of the total non-adaptive nodes) as reported in Section 4 represents an acceleration of about 90 times with respect to the non-adaptive case.

\section{Lagrangian dynamic SGS model}

The main objective of the current work is to develop a local SGS model for SCALES of inhomogeneous turbulent flows. One way to achieve this goal would be to use the

* $N$ is also the number of grid points due to one-to-one correspondence between wavelet locations and grid points. 
local Smagorinsky model based on a modified Germano dynamic procedure, redefined in terms of the wavelet thresholding filters introduced in [4]. However, as in the classical LES approach, e.g., [15], the local formulation would lead to numerical instability. The volume averaging procedure, introduced in [4] to stabilize the solution, leads to the definition of a global (spatially non-variable) coefficient. This limits the applicability of the model to flows with at least one homogeneous direction.

We now extend the Lagrangian dynamic path-line averaging approach of [6] and apply it to SCALES. Following [4], where the SGS stresses (5) were shown to scale like $\epsilon^{2}$, the deviatoric part (hereafter noted with a star) of the SGS stress tensor is approximated by the Smagorinsky eddy-viscosity model

$$
\tau_{i j}^{*} \cong-2 C_{S} \Delta^{2} \epsilon^{2}\left|\bar{S}^{>\epsilon}\right| \bar{S}_{i j}^{>\epsilon},
$$

where ${\overline{S_{i j}}}^{>\epsilon}=1 / 2\left(\partial{\overline{u_{i}}}^{>\epsilon} / \partial x_{j}+\partial{\overline{u_{j}}}^{>\epsilon} / \partial x_{i}\right)$ is the resolved rate-of-strain tensor, $\left|\bar{S}^{>\epsilon}\right|=\left(2{\overline{S_{i j}}}^{>\epsilon}{\overline{S_{i j}}}^{>\epsilon}\right)^{1 / 2}$, and $\Delta(\mathbf{x}, t)$ is the local characteristic filter length-scale. The latter is the key-parameter in the SCALES formulation as it strictly reflects the adaptive nature of the method. Once a wavelet threshold is given, the corresponding thresholding mask implicitly defines a point-wise time-dependent filter width. This is different from classical LES where the local, possibly non-uniform, filter width is defined a priori and does not depend on the actual flow realization.

Following the modified dynamic procedure of [4], the residual stress tensor at the test-filter level is defined as

$$
T_{i j}={\overline{u_{i} u_{j}}}^{2 \epsilon}-{\overline{u_{i}}}^{>2 \epsilon}{\overline{u_{j}}}^{>2 \epsilon},
$$

where $\overline{(\cdot)}>2 \epsilon$ corresponds to the wavelet test filter at twice the threshold, i.e. the test filter is defined by Eq. (2) with $\epsilon$ replaced by $2 \epsilon$. Since the wavelet filter is a projection operator it satisfies $\overline{\overline{(\cdot)}}^{>\epsilon} \equiv \overline{(\cdot)}^{>2 \epsilon}$. Therefore, by filtering (5) at the test filter level and combining it with (7), the following modified Germano identity for the Leonard stresses is obtained:

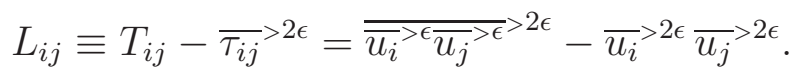

Exploiting the model (6) and the analogous relation for the test filtered SGS stresses

$$
T_{i j}^{*} \cong-2 C_{S} \Delta^{2}(2 \epsilon)^{2}\left|\bar{S}^{>2 \epsilon}\right|{\overline{S_{i j}}}^{>2 \epsilon},
$$

one obtains

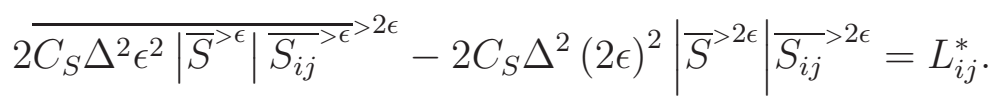

As in classical dynamic LES, a least square solution to (10) leads to the following local Smagorinsky model coefficient definition:

$$
C_{S}(\mathbf{x}, t) \epsilon^{2}=\frac{L_{i j}^{*} M_{i j}}{M_{h k} M_{h k}},
$$

where

$$
M_{i j} \equiv 2 \Delta^{2}\left[{\overline{\left|\bar{S}^{>\epsilon}\right| \bar{S}_{i j}}}^{>\epsilon}-4\left|\bar{S}^{>2 \epsilon}\right|{\overline{S_{i j}}}^{>2 \epsilon}\right]
$$


The coefficient $C_{S}$ can be actually positive or negative, which allows for local backscatter of energy from unresolved to resolved modes. However, it has been found that negative values of $C_{S}$ cause numerical instabilities. To avoid this, the Lagrangian dynamic model formulation of [6] is exploited by taking the following statistical averages along the trajectory of a fluid particle:

$$
\begin{aligned}
& \mathcal{I}_{L M}(\boldsymbol{x}, t)=\frac{1}{T} \int_{-\infty}^{t} e^{\frac{\tau-t}{T}} L_{i j}(\boldsymbol{x}(\tau), \tau) M_{i j}(\boldsymbol{x}(\tau), \tau) d \tau \\
& \mathcal{I}_{M M}(\boldsymbol{x}, t)=\frac{1}{T} \int_{-\infty}^{t} e^{\frac{\tau-t}{T}} M_{h k}(\boldsymbol{x}(\tau), \tau) M_{h k}(\boldsymbol{x}(\tau), \tau) d \tau
\end{aligned}
$$

This leads to the definition of the local Smagorinsky model coefficient:

$$
C_{S}(\mathbf{x}, t) \epsilon^{2}=\frac{\mathcal{I}_{L M}}{\mathcal{I}_{M M}}
$$

To avoid the computationally expensive procedure of Lagrangian path-line averaging, (13) and (14) are differentiated with respect to time leading to the evolution equations for $\mathcal{I}_{L M}$ and $\mathcal{I}_{M M}$ :

$$
\begin{aligned}
& \frac{\partial \mathcal{I}_{L M}}{\partial t}+\bar{u}_{m}^{>\epsilon} \frac{\partial \mathcal{I}_{L M}}{\partial x_{m}}=\frac{1}{T}\left(L_{i j} M_{i j}-\mathcal{I}_{L M}\right), \\
& \frac{\partial \mathcal{I}_{M M}}{\partial t}+\bar{u}_{m}^{>\epsilon} \frac{\partial \mathcal{I}_{M M}}{\partial x_{m}}=\frac{1}{T}\left(M_{h k} M_{h k}-\mathcal{I}_{M M}\right) .
\end{aligned}
$$

The relaxation time scale $T$ is defined as $T(\mathbf{x}, t)=\theta \Delta\left(\mathcal{I}_{L M} \mathcal{I}_{M M}\right)^{-1 / 8}$, where $\theta$ is a dimensionless parameter of order unity, which is one of the options suggested by [6].

Equations (16) and (17) should be solved together with the governing equations, (3) and (4), resulting in the availability of the local Smagorinsky model coefficient at every time step. It should be noted that both $\mathcal{I}_{L M}$ and $\mathcal{I}_{M M}$ have higher frequency content when compared to the velocity field. This is due to two main factors: the quartic character of nonlinearity of $\mathcal{I}_{L M}$ and $\mathcal{I}_{M M}$ with respect to velocity and the creation of small scales due to chaotic convective mixing. One way to deal with this problem is to use the original formulation of Meneveau et al. [6] that does not solve equations (16) and (17) directly. Instead the linearized version of characteristics is used to solve Eqs. (16) and (17), which introduces sufficient numerical diffusion that no stabilization is necessary. Since AWCM uses high order central difference scheme for derivative operators, the resulting numerical approach does not have numerical diffusion and in order to adequately resolve both $\mathcal{I}_{L M}$ and $\mathcal{I}_{M M}$, one would need to have a substantially finer computational mesh than the one required by the velocity field, which is impractical. To by-pass this problem we consider two different extensions of the original Lagrangian path-line averaging procedure: Lagrangian path-tube averaging and Lagrangian path-line diffusive averaging.

The Lagrangian path-tube averaging consists of taking the statistically filtered averages over the trajectory of a fluid particle:

$$
\mathcal{I}_{L M}(\boldsymbol{x}, t)=\frac{1}{T} \int_{-\infty}^{t} \iiint_{D} e^{\frac{\tau-t}{T}} G(\boldsymbol{y}-\boldsymbol{x}(\tau), \boldsymbol{x}(\tau)) L_{i j}(\boldsymbol{y}, \tau) M_{i j}(\boldsymbol{y}, \tau) d \tau d \boldsymbol{y}
$$




$$
\mathcal{I}_{M M}(\boldsymbol{x}, t)=\frac{1}{T} \int_{-\infty}^{t} \iiint_{D} e^{\frac{\tau-t}{T}} G(\boldsymbol{y}-\boldsymbol{x}(\tau), \boldsymbol{x}(\tau)) M_{h k}(\boldsymbol{y}, \tau) M_{h k}(\boldsymbol{y}, \tau) d \tau d \boldsymbol{y}
$$

where $G(\boldsymbol{\xi}, \boldsymbol{x})$ is the local, location dependent, low-pass filter with the second variable denoting filter location. Note that the low-pass filter averages the values in the neighborhood of the path-line, effectively making it path-tube averaging. Also note that if $G(\boldsymbol{\xi}, \boldsymbol{x})=1$, i.e. no additional spatial filter is applied, the formulations are identical to the one in [6]. This modified averaging procedure leads to the following evolution equations for the auxiliary variables $\mathcal{I}_{L M}$ and $\mathcal{I}_{M M}$ :

$$
\begin{aligned}
& \frac{\partial \mathcal{I}_{L M}}{\partial t}+\bar{u}_{l}^{>\epsilon} \frac{\partial \mathcal{I}_{L M}}{\partial x_{l}}=\frac{1}{T}\left({\overline{L_{i j} M_{i j}}}^{\mathrm{LP}}-\mathcal{I}_{L M}\right), \\
& \frac{\partial \mathcal{I}_{M M}}{\partial t}+\bar{u}_{m}^{>\epsilon} \frac{\partial \mathcal{I}_{M M}}{\partial x_{m}}=\frac{1}{T}\left({\overline{M_{h k} M_{h k}}}^{\mathrm{LP}}-\mathcal{I}_{M M}\right),
\end{aligned}
$$

where $\overline{(\cdot)}$ LP denotes low-pass filtering based on $G(\boldsymbol{\xi}, \boldsymbol{x})$.

In the Lagrangian path-line diffusive averaging approach an additional artificial diffusion term is added to the evolution equations:

$$
\begin{aligned}
& \frac{\partial \mathcal{I}_{L M}}{\partial t}+\bar{u}_{l}^{>\epsilon} \frac{\partial \mathcal{I}_{L M}}{\partial x_{l}}=\frac{1}{T}\left(L_{i j} M_{i j}-\mathcal{I}_{L M}\right)+\mathcal{D}_{\mathcal{I}} \frac{\partial^{2} \mathcal{I}_{L M}}{\partial x_{l} \partial x_{l}}, \\
& \frac{\partial \mathcal{I}_{M M}}{\partial t}+\bar{u}_{m}^{>\epsilon} \frac{\partial \mathcal{I}_{M M}}{\partial x_{m}}=\frac{1}{T}\left(M_{h k} M_{h k}-\mathcal{I}_{M M}\right)+\mathcal{D}_{\mathcal{I}} \frac{\partial^{2} \mathcal{I}_{M M}}{\partial x_{m} \partial x_{m}} .
\end{aligned}
$$

To avoid the creation of small scales, the diffusion time scale, $\Delta^{2} / \mathcal{D}_{\mathcal{I}}$, should be smaller than the convective time scale associated with local strain, i.e. $\left|\bar{S}^{>\epsilon}\right|^{-1}$, which results in $\mathcal{D}_{\mathcal{I}}=C_{\mathcal{I}} \Delta^{2}\left|\bar{S}^{>\epsilon}\right|$, where $C_{\mathcal{I}}$ is a dimensionless parameter of order unity. Note that the Lagrangian path-line diffusive averaging procedure can be formally derived by using differential implicit filters described in Appendix A. Substituting the implicit integrodifferential form (A.11) of the Lagrangian path-tube averaging into Eqs. (18) and (19) and assuming, for simplicity, $\mathcal{D}_{\mathcal{I}_{L M}}=\mathcal{D}_{\mathcal{I}_{M M}}=\mathcal{D}_{\mathcal{I}}$ one can derive Eqs. (22) and (23).

Combining these two approaches results in the Lagrangian path-line/tube diffusive averaged equations:

$$
\begin{aligned}
& \frac{\partial \mathcal{I}_{L M}}{\partial t}+\bar{u}_{l}^{>\epsilon} \frac{\partial \mathcal{I}_{L M}}{\partial x_{l}}=\frac{1}{T}\left({\overline{L_{i j} M_{i j}}}^{\mathrm{LP}}-\mathcal{I}_{L M}\right)+\mathcal{D}_{\mathcal{I}} \frac{\partial^{2} \mathcal{I}_{L M}}{\partial x_{l} \partial x_{l}}, \\
& \frac{\partial \mathcal{I}_{M M}}{\partial t}+\bar{u}_{m}^{>\epsilon} \frac{\partial \mathcal{I}_{M M}}{\partial x_{m}}=\frac{1}{T}\left({\overline{M_{h k} M_{h k}}}^{\mathrm{LP}}-\mathcal{I}_{M M}\right)+\mathcal{D}_{\mathcal{I}} \frac{\partial^{2} \mathcal{I}_{M M}}{\partial x_{m} \partial x_{m}} .
\end{aligned}
$$

Note that the case $G=1$ and $C_{\mathcal{I}}=0$ is equivalent to the original Lagrangian formulation of $[6]$.

\section{Results}

In this section we apply the proposed SCALES model to incompressible isotropic decaying turbulence. Though the proposed localized models are specifically designed to simulate complex inhomogeneous turbulent flows, it is nevertheless enlightening to test them for a case for which well known theoretical and experimental results exist. 


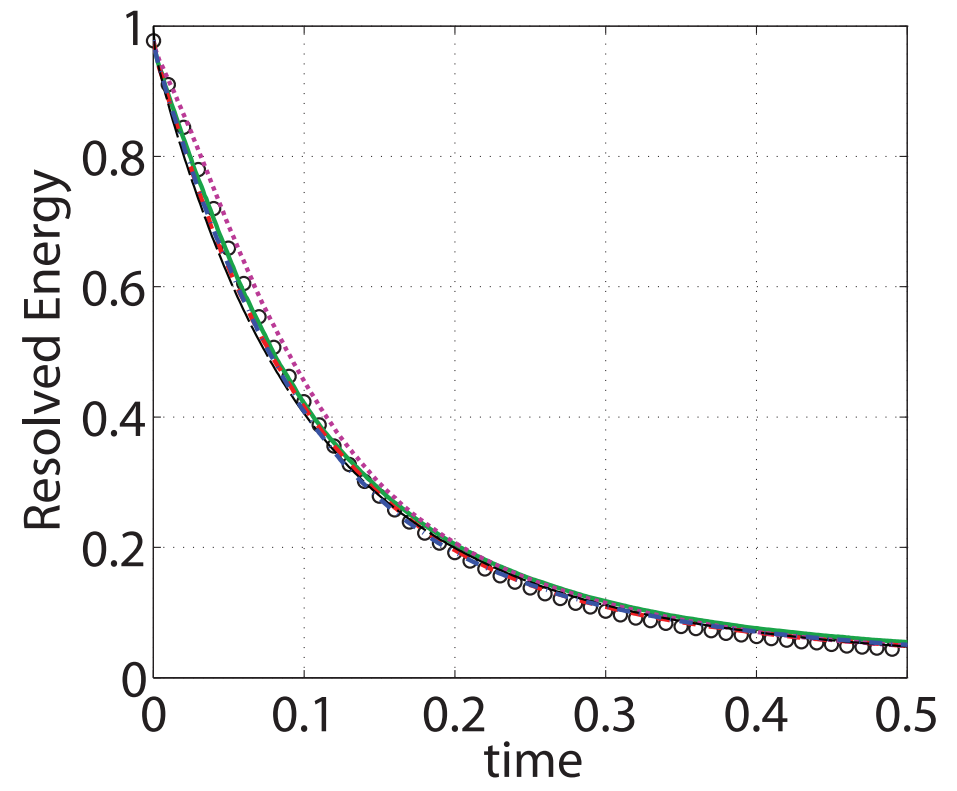

Figure 2. Energy decay for SCALES with the Lagrangian path-line diffusive (---- ) and path-tube averaging (----- ) models, SCALES with global dynamic model (— ), SCALES with no model $(\cdots \cdots \cdots)$, reference LES with global dynamic model (- - ), and wavelet filtered DNS (o).

In addition, the homogeneous turbulence case allows a detailed comparison with the existing reference solutions for DNS, LES, and SCALES (with global dynamic model). Moreover, decaying turbulence is a challenging example of statistically unsteady flow that stands as a good test-case for a-posteriori verifying the accuracy of the proposed SGS stress model. The initial velocity field is a realization of a statistically stationary turbulent flow at $\operatorname{Re}_{\lambda}=72$ ( $\lambda$ being the Taylor microscale) that is provided by a fully de-aliased pseudo-spectral DNS with $128^{3}$ Fourier modes [5]. Due to the finite difference nature of the AWCM solver, the initial SCALES resolution in each direction must be doubled in order to retain the spectral energy content. In other words, SCALES is run using a maximum resolution corresponding to $256^{3}$ grid points. Note that due to the nature of the decaying turbulence, $256^{3}$ resolution is only required during initial times with gradual decrease of the maximum level of resolution as turbulence kinetic energy decays and Taylor microscale Reynolds number decreases.

The choice of the thresholding parameter, $\epsilon$, in (2) is somewhat arbitrary: the smaller it is, the weaker the SGS dissipation is, with SCALES approaching Coherent Vortex Simulation [16, 4] and wavelet-based DNS for even smaller values of $\epsilon \leq 10^{-3}$. On the other hand, when $\epsilon$ is too large, too many modes are discarded and the energy cascade is no longer captured. All the SCALES results reported in this paper have been obtained using the wavelet thresholding parameter $\epsilon=0.43$ as a compromise between these limits.

The Lagrangian local modelling variables are initialized as $\mathcal{I}_{M M}=M_{h k} M_{h k}$ and 


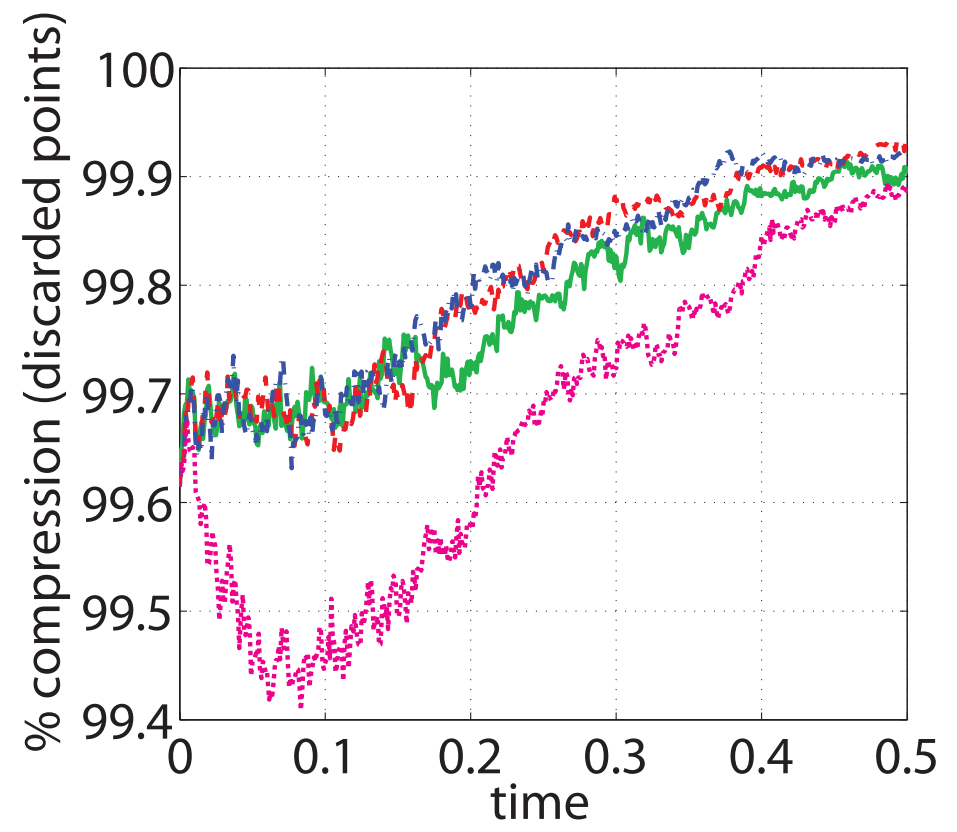

Figure 3. Field compression: SCALES with the Lagrangian path-line diffusive (---- ) and path-tube averaging (----- ) models, SCALES with global dynamic model

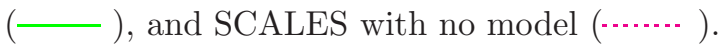

$\mathcal{I}_{L M}=\bar{C}_{s} \epsilon^{2} \mathcal{I}_{M M}$, where $\bar{C}_{s}$ is the volume averaged Smagorinsky model coefficient (11). For the relaxation time scale, the value $\theta=0.75$ suggested by $[6]$ is chosen. For Lagrangian path-line diffusive averaging, a diffusion coefficient $C_{\mathcal{I}} \geq 5$ has been found to produce acceptable results while approaching the global dynamic model for very large values of the coefficient. In the case of Lagrangian path-tube averaging, the same stabilizing effect is obtained by means of local volume averaging along the path-line, provided that the linear cross-sectional dimensions of the path-tube are not smaller than the local characteristic filter width, $\Delta$.

In figures 2-4 the resolved kinetic energy decay, grid compression (percentage of the discarded grid points), and modelled SGS dissipation (percentage of the total dissipation) for proposed SCALES are compared to: a-posteriori wavelet filtered DNS, classical LES, SCALES with global dynamic model, and SCALES with no model. The LES is performed using the non-adaptive wavelet collocation solver on a regular $64^{3}$ grid. The solution is de-aliased by performing a wavelet transform on the velocity field and zeroing the highest level wavelet coefficients for each time step. As to figure 2, the resolved kinetic energy is normalized with respect to the initial unfiltered DNS energy content. The grid compression is evaluated with respect to the maximum field resolution. The time scale used to report the results corresponds to approximately ten initial eddy-turnover times.

As can be seen from the energy decay plot in figure 2, the case of SCALES with no model is only slightly under-dissipative. From figure 5 we see that this case accurately captures the energy and enstrophy spectra. By examining the grid compression reported in figure 3 we can see that due to the adaptive nature of the numerical algorithm, the 


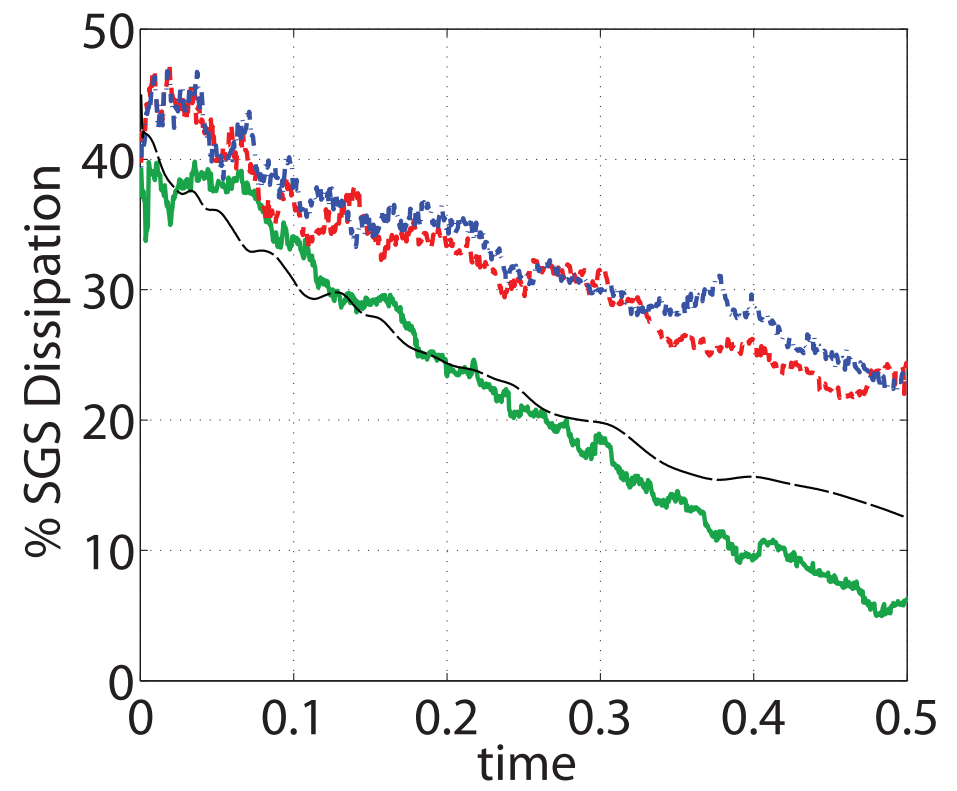

Figure 4. Percentage of SGS (modelled) dissipation: SCALES with the Lagrangian path-line diffusive (---- ) and path-tube averaging (----- ) models, SCALES with global dynamic model ( - ), and reference LES with global dynamic model ( - - $)$.

absence of SGS dissipation results in energy transfer to the small scales, where the energy is dissipated by viscous stresses. This process results in an increase in the number of the degrees of freedom and causes the solution to approach CVS [4]. This effect would be more pronounced for higher Reynolds number flows, since SCALES with no model would have to resolve all the scales up to Kolmogorov scale and would rely on molecular viscosity as the only dissipative mechanism. The adaptive nature of the AWCM makes comparison of simulations tricky because the algorithm itself attempts to add resolution if the physical problem is under resolved. In this case good results are obtained at the cost of increased resolution. Another interesting observation is that despite the similar initial compression, the local Lagrangian models show a higher level of SGS dissipation because they capture the local structure of the flow, rather than simply providing the necessary mean energy dissipation (as in the case of both the global dynamic model and LES). This, in turn, results in higher compression for larger times. It worth noting that the level of SGS dissipation of SCALES closely matches that of the global model and reference LES.

Another crucial feature of the SCALES approach is seen in the energy and, more importantly, enstrophy spectra, which are shown respectively in figures 5 and 6 for two different times. In contrast to classical LES, the SCALES results match not only in terms of temporal evolution of the total resolved turbulent kinetic energy, but also in terms of the DNS energy and enstrophy density spectra up to the dissipative wavenumber range. It is important to emphasize that this close match is achieved using less than $0.4 \%$ of the total non-adaptive nodes required for a DNS with the same wavelet solver. 

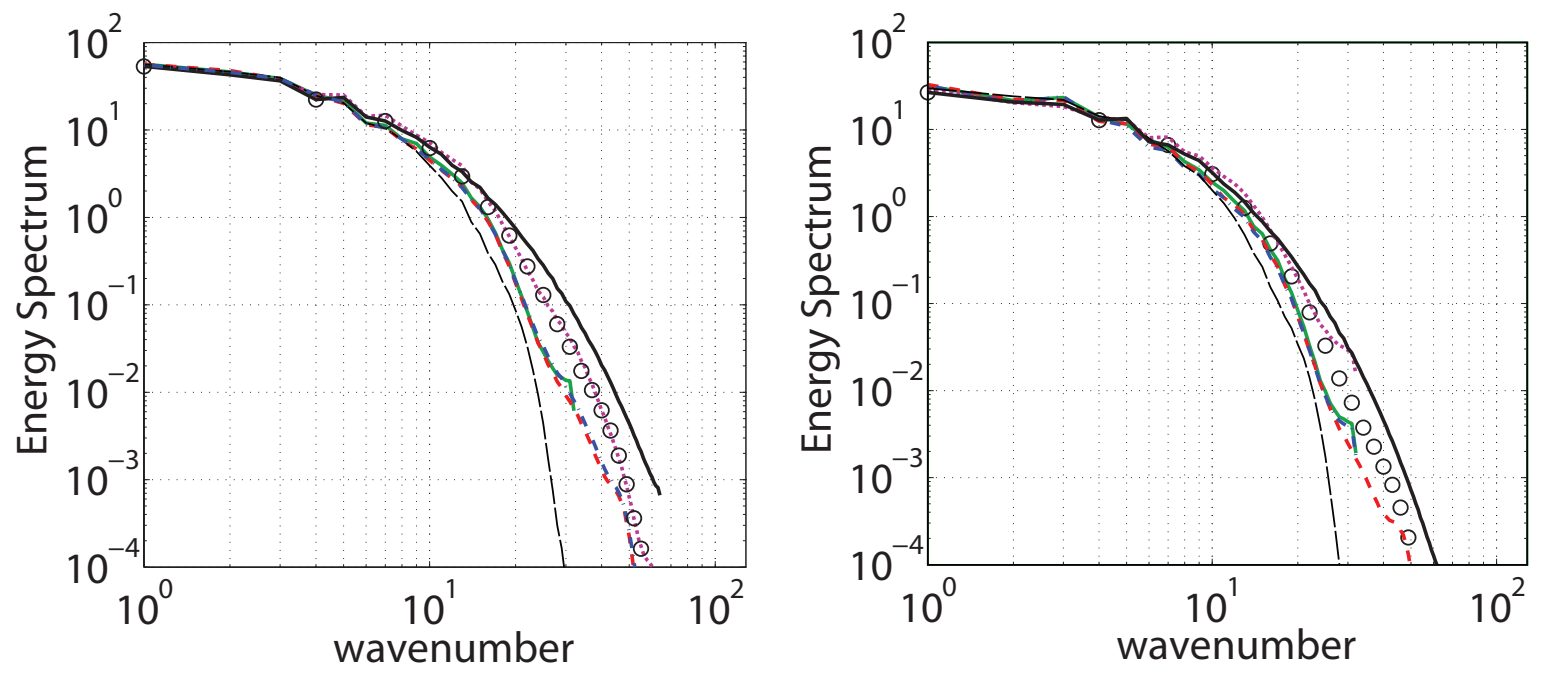

Figure 5. Energy density spectra at $t=0.08$ (left) and $t=0.16$ (right): SCALES with the Lagrangian path-line diffusive (---- ) and path-tube averaging (-.-.- ) models, SCALES with global dynamic model ( - ), SCALES with no model ( - …... ), spectral DNS ( $(\longrightarrow)$, wavelet filtered DNS (o), and reference LES with global dynamic model (- - ).

To highlight the significance of such a close match, it is interesting to compare these results with those of an LES with the global dynamic Smagorinsky model. Despite the fact that LES uses almost four times the number of modes $(1.56 \%)$, it fails to capture the small-scale features of the spectrum. In addition, the total resolved LES kinetic energy is noticeably below the filtered DNS curve for moderate and high wavenumbers. These differences are even more pronounced for the enstrophy spectra.

It is worth stressing the unique feature of the SCALES approach, nameley the coupling of modelled SGS dissipation to grid compression: more grid points are used for models with lower levels of SGS dissipation. In other words, the SCALES approach compensates for inadequate SGS dissipation by increasing the local resolution and, hence, the level of resolved viscous dissipation. This can be seen clearly by comparing to the SCALES results with no SGS model.

As stated earlier, the absence of SGS dissipation results in the transfer of energy from low to high wavenumbers, filling the entire wavenumber range, bringing the energy and enstrophy spectra close to the wavelet filtered DNS spectrum. This processes continues until the lack of SGS dissipation is balanced by the viscous dissipation. Recall that enstrophy and viscous dissipation spectra are identical if properly normalized. Thus, an accurate enstrophy spectrum ensures proper viscous dissipation. On the other hand, the increase of energy in high wavenumber range results in an increase of degrees of freedom (active wavelet coefficients or grid points), as seen in figure 3. The energy and enstrophy spectra for SCALES with local Lagrangian models closely match each other and agree reasonably well with the spectra for filtered DNS. The non-local character of the dissipation of the global dynamic model results in over-dissipation at small scales and, subsequently, smaller wavelet coefficients on the finest level of resolution, which 

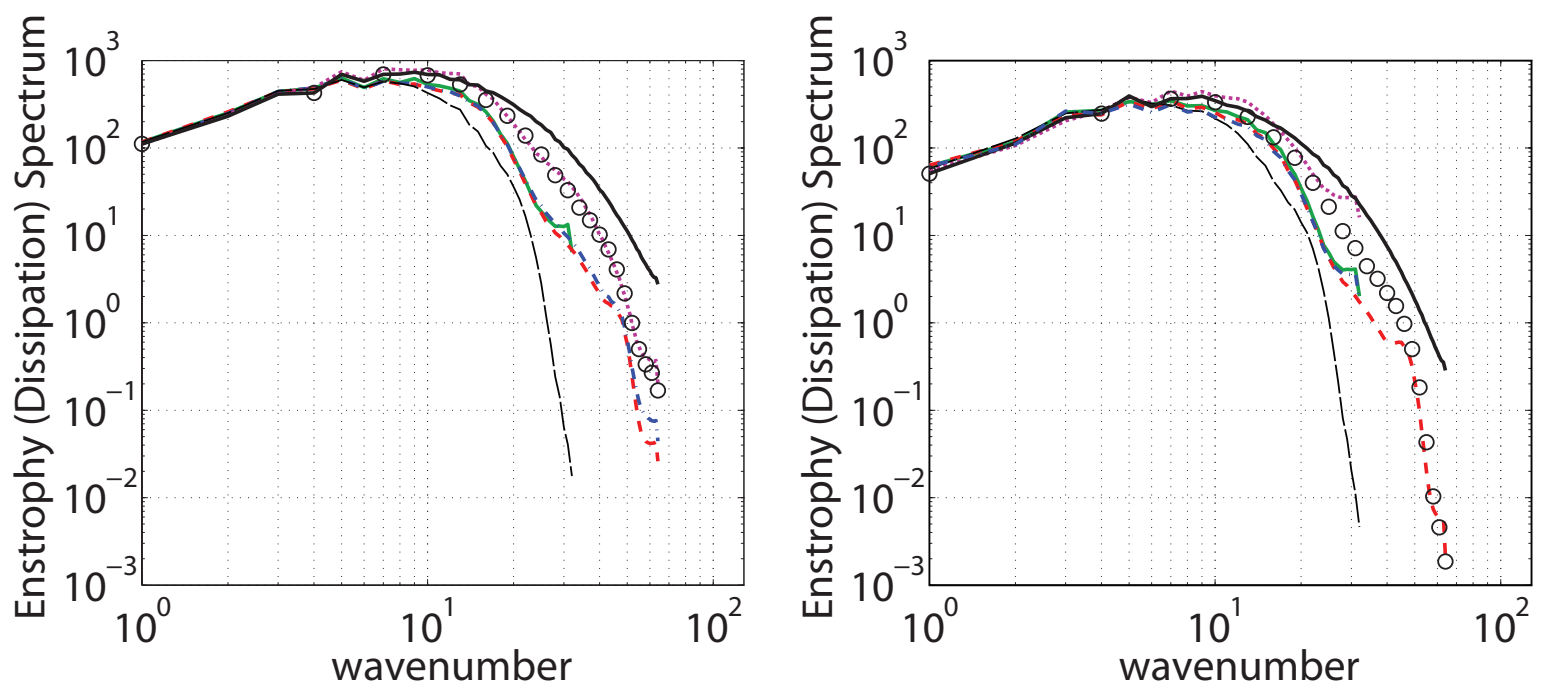

Figure 6. Enstrophy density spectra at $t=0.08$ (left) and $t=0.16$ (right): SCALES with the Lagrangian path-line diffusive (---- ) and path-tube averaging (-.-- ) models, SCALES with global dynamic model (— $)$, SCALES with no model (……), spectral DNS ( $(\longrightarrow)$, wavelet filtered DNS (o), and reference LES with global dynamic model ( $-\longrightarrow)$.

ultimately results in the earlier removal of the finest level of resolution from the adaptive computational grid.

Finally, SCALES with the local dynamic Smagorinsky model with both types of Lagrangian averaging are virtually identical, which highlights the similarities of both averaging approaches.

\section{Conclusions}

The development of local SGS models is unavoidable for the application of SCALES to practical engineering flows. In this work, new local SCALES models based on Lagrangian path-line diffusive and path-tube averaging are developed and assessed in terms of accuracy and efficiency. The results in this work show that localized Lagrangian dynamic SGS models can be successfully used with the SCALES approach. Further studies for strongly inhomogeneous higher Reynolds number flows are currently underway and will be reported in a future publication.

\section{Acknowledgements}

This work was supported by the Department of Energy (DOE) under Grant No. DEFG02-05ER25667, the National Science Foundation (NSF) under grants No. EAR0327269 and ACI-0242457, and the National Aeronautics and Space Administration (NASA) under grant No. NAG-1-02116. In addition G. De Stefano was partially supported by a grant from Regione Campania (LR 28/5/02 n.5). N. K.-R. Kevlahan was supported by the Natural Sciences and Engineering Research Council of Canada. 
The authors also thank Profs. Charles Meneveau and Thomas S. Lund for helpful suggestions.

\section{Appendix A. Differential Filters}

The Lagrangian path-line diffusive averaging approach can be also derived by using differential filters. To illustrate the derivation, for simplicity let us consider onedimensional spatial filtering

$$
\bar{\phi}^{\mathrm{LP}}(x, t)=\int_{D} G(y-x, x) \phi(y, t) d y .
$$

Performing Taylor series expansion of $\phi(y, t)$ around $x$ yields

$$
\phi(y, t)=\sum_{k=0}^{+\infty} \frac{(y-x)^{k}}{k !} \frac{\partial^{k} \phi}{\partial x^{k}}(x, t) .
$$

Substituting Eq. (A.2) into Eq. (A.1) and changing the order of integration and summation one can obtain

$$
\bar{\phi}^{\mathrm{LP}}(x, t)=\sum_{k=0}^{+\infty}\left(\int_{D} \frac{(y-x)^{k}}{k !} G(y-x, x) d y\right) \frac{\partial^{k} \phi}{\partial x^{k}}(x, t) .
$$

Denoting the term in parenthesis as differential filter coefficient $\mathcal{M}_{k}$ and taking into account that $\mathcal{M}_{0}=1$, filter (A.1) can be rewritten as an explicit differential filter

$$
\bar{\phi}^{\mathrm{LP}}(x, t)=\phi(x, t)+\sum_{k=1}^{+\infty} \mathcal{M}_{k} \frac{\partial^{k} \phi}{\partial x^{k}}(x, t) \text {. }
$$

This infinite series can be inverted and the differential filter can be rewritten in implicit (Pade) differential form

$$
\bar{\phi}^{\mathrm{LP}}(x, t)=\phi(x, t)+\sum_{k=1}^{+\infty} \mathcal{D}_{k} \frac{\partial^{k} \bar{\phi}^{\mathrm{LP}}}{\partial x^{k}}(x, t),
$$

where $\mathcal{D}_{k}$ are the implicit differential filter coefficients. This analysis can be easily extended to multiple dimensions, e.g. $[8,9,10]$. Note that since the first moments for most of the low-pass filters are always zero, the simplest inhomogeneous implicit multi-dimensional differential filter is given by

$$
\bar{\phi}^{\mathrm{LP}}(\boldsymbol{x}, t)=\phi(\boldsymbol{x}, t)+\mathcal{D}_{2}(\boldsymbol{x}, t) \frac{\partial^{2} \bar{\phi}^{\mathrm{LP}}}{\partial x_{k} \partial x_{k}}(\boldsymbol{x}, t),
$$

where repeated indices imply the summation.

The Lagrangian path-tube averaging along the trajectory of a fluid particle, $\boldsymbol{x}(t)$, for the field variable $\phi(\boldsymbol{x}, t)$ can be written as

$$
\mathcal{I}_{\phi}(\boldsymbol{x}, t)=\frac{1}{T} \int_{-\infty}^{t} \iiint_{D} e^{\frac{\tau-t}{T}} G(\boldsymbol{y}-\boldsymbol{x}(\tau), \boldsymbol{x}(\tau)) \phi(\boldsymbol{y}, \tau) d \tau d \boldsymbol{y}
$$


or using low-pass filter notation as

$$
\mathcal{I}_{\phi}(\boldsymbol{x}, t)=\frac{1}{T} \int_{-\infty}^{t} e^{\frac{\tau-t}{T}} \bar{\phi}^{\mathrm{LP}}(\boldsymbol{x}(\tau), \tau) d \tau .
$$

Substituting inhomogeneous differential implicit filter (A.6) into (A.8) yields

$$
\mathcal{I}_{\phi}(\boldsymbol{x}, t)=\frac{1}{T} \int_{-\infty}^{t} e^{\frac{\tau-t}{T}}\left(\phi(\boldsymbol{x}(\tau), \tau)+\mathcal{D}_{2}(\boldsymbol{x}(\tau), \tau) \frac{\partial^{2} \bar{\phi}^{\mathrm{LP}}}{\partial x_{k} \partial x_{k}}(\boldsymbol{x}(\tau), \tau)\right) d \tau
$$

The deconvoluted implicit filter coefficient $\mathcal{D}_{\mathcal{I}_{\phi}}$ can be introduced using the following definition:

$$
\left.\int_{-\infty}^{t} e^{\frac{\tau-t}{T}} \mathcal{D}_{\mathcal{I}_{\phi}}(\boldsymbol{x}(\tau), \tau) \frac{\partial^{2} \mathcal{I}_{\phi}}{\partial x_{k} \partial x_{k}}\right|_{\boldsymbol{x}(\tau), \tau} d \tau=\left.\int_{-\infty}^{t} e^{\frac{\tau-t}{T}} \mathcal{D}_{2}(\boldsymbol{x}(\tau), \tau) \frac{\partial^{2} \bar{\phi}}{\partial x_{k} \partial x_{k}}\right|_{\boldsymbol{x}(\tau), \tau} d \tau \text {. }
$$

Finally, with this definition Eq. (A.9) can be rewritten in the following implicit integrodifferential form:

$$
\mathcal{I}_{\phi}(\boldsymbol{x}, t)=\frac{1}{T} \int_{-\infty}^{t} e^{\frac{\tau-t}{T}}\left(\phi(\boldsymbol{x}(\tau), \tau)+\mathcal{D}_{\mathcal{I}_{\phi}}(\boldsymbol{x}(\tau), \tau) \frac{\partial^{2} \mathcal{I}_{\phi}}{\partial x_{k} \partial x_{k}}(\boldsymbol{x}(\tau), \tau)\right) d \tau \text {. }
$$

\section{References}

[1] P. Moin. Advances in large eddy simulation methodology of complex flows. Int. J. Heat Fluid Flow, 23, 710-720, 2002.

[2] S. B. Pope. Ten questions concerning the large-eddy simulation of turbulent flows. New J. Phys, 6(35), 2004.

[3] D. E. Goldstein and O. V. Vasilyev. Stochastic coherent adaptive large eddy simulation method. Phys. Fluids, 16(7), 2497-2513, 2004.

[4] D.E. Goldstein, O.V. Vasilyev, and N.K.-R. Kevlahan. CVS and SCALES simulation of 3D isotropic turbulences. J. of Turbulence, 6(37), 1-20, 2005.

[5] G. De Stefano, D. E. Goldstein, and O. V. Vasilyev. On the role of sub-grid scale coherent modes in large eddy simulation. Journal of Fluid Mechanics, 525, 263-274, 2005.

[6] C. Meneveau, T. S. Lund, and W. H. Cabot. A Lagrangian dynamic subgrid-scale model of turbulence. J. Fluid Mech., 319, 353-385, 1996.

[7] I. Daubechies. Ten Lectures on Wavelets. Number 61 in CBMS-NSF Series in Applied Mathematics. SIAM, Philadelphia, 1992.

[8] O. V. Vasilyev, T. S. Lund, and P. Moin. A general class of commutative filters for LES in complex geometries. J. Comp. Phys., 146, 105-123, 1998.

[9] A. L. Marsden, O. V. Vasilyev, and P. Moin. Construction of commutative filters for LES on unstructured meshes. J. Comp. Phys., 175, 584-603, 2002.

[10] A. Haselbacher and O. V. Vasilyev. Commutative discrete filtering on unstructured grids based on least-squares techniques. J. Comp. Phys., 187(1), 197-211, 2003.

[11] O. V. Vasilyev. Solving multi-dimensional evolution problems with localized structures using second generation wavelets. Int. J. Comp. Fluid Dyn., Special issue on Highresolution methods in Computational Fluid Dynamics, 17(2), 151-168, 2003. 
[12] O. V. Vasilyev and C. Bowman. Second generation wavelet collocation method for the solution of partial differential equations. J. Comp. Phys., 165, 660-693, 2000.

[13] O. V. Vasilyev and N. K.-R. Kevlahan. An adaptive multilevel wavelet collocation method for elliptic problems. J. Comp. Phys., 206(2), 412-431, 2005.

[14] W. Sweldens. The lifting scheme: A construction of second generation wavelets. SIAM J. Math. Anal., 29(2), 511-546, 1998.

[15] M. Germano, U. Piomelli, P. Moin, and W.H. Cabot. A dynamic subgrid-scale eddy viscosity model. Phys. Fluids A, 3(7), 1760-1765, 1991.

[16] M. Farge, K. Schneider, and N. Kevlahan. Non-Gaussianity and coherent vortex simulation for two-dimensional turbulence using an adaptive orthogonal wavelet basis. Phys. Fluids., 11(8), 2187-2201, 1999. 